\title{
Möller, Rainer / Sajak, Clauß Peter (Hg.) (2020). Religionspädagogik für Erzieherinnen. Ein ökumenisches Arbeitsbuch. Stuttgart: Kohlhammer. ISBN 978-3-I 7-036420-2. 274 Seiten.
}

\author{
Katrin Petri \\ Kommunales Integrationszentrum Gelsenkirchen (katrinpetri@yahoo.com)
}

Das Arbeitsbuch Religionspädagogik für Erzieher*innen ist eine Neubearbeitung des mehrfach aufgelegten gleichnamigen Arbeitsbuches von Rainer Möller und Reinmar Tschirch. Neu ist die Erarbeitung in einem interkonfessionellen Autorenteam, was den Einsatz des Werkes sowohl im konfessionell getrennten als auch im konfessionellkooperativen oder konfessionell gemischten Kontext erlaubt. Darüber hinaus bietet das Handbuch an vereinzelten Stellen immer wieder auch Anregungen, eine interreligiöse sowie intrareligiöse Perspektive miteinzubeziehen. Aufbau und Struktur des Arbeitsbuches orientieren sich an den benötigten religionspädagogischen Kompetenzen von Erzieher*innen für die Praxis in den Kindertageseinrichtungen.

Im ersten Kapitel geht es um Professionalität und Berufsrolle von Erzieher*innen im religionspädagogischen Kontext. Dieter Miedza stellt in diesem Zusammenhang ein Kompetenzmodell vor, das sowohl personale Kompetenzen (Selbstreflexionskompetenz, Innovationskompetenz, Beziehungskompetenz, Handlungskompetenz) als auch theologisch-didaktische Grundkompetenzen (religiöses Grundwissen, religionskundliches Orientierungswissen, didaktisches Vermittlungswissen, methodisches Gestaltungswissen) berücksichtigt und sinnvoll miteinander in Beziehung setzt. Zeitgemäß erscheint insbesondere die offene Haltung in Bezug auf intra- und interreligiöse Diversität sowohl auf Seiten des Fachpersonals als auch in der Kommunikation und Zusammenarbeit mit den Familien. Die Verknüpfung zum Habitus-Modell sensibilisiert in diesem Zusammenhang für interkontextuelle Abhängigkeiten psychischer, sozialer, kognitiver und physischer Art, die unser Denken und Handeln jeweils beeinflussen.

Das zweite Kapitel widmet sich dem Kind und seiner religiösen Entwicklung. Hier werden in erster Linie Stufenmodelle zur kognitiven, moralischen und religiösen Entwicklung (Piaget, Kohlberg, Goldman, Oser \& Gmünder, Kuld) eingeführt. Obwohl gegen Ende des einführenden Kapitels auch eine kritische Perspektive in Bezug auf Stufenmodelle aufgeworfen wird, bleibt hier ein Einbezug wissenschaftlicher Erkenntnisse der neueren Kognitionspsychologie z. B. in Bezug auf eine anregungsabhängige domänenspezifische Kompetenzentwicklung aus.

Kapitel drei behandelt das Thema Religion und religiöse Identität in der modernen Gesellschaft. Über einen einführenden Diskurs zu verschiedenen Definitionsansätzen des Religionsbegriffes, anthropologisch in Anlehnung an Schleiermacher und Tillich, funktional wie z. B. bei Kaufmann, Weber, Lübbe, Luhmann und Durkheim oder religionssoziologisch dimensional wie im Fünf-Dimensionenmodell nach Glock, werden im Anschluss soziokulturelle Tendenzen einer postmodernen Lebenswelt nachgezeichnet (Individualisierung, Globalisierung, Pluralisierung, Privatisierung) und ihre Konsequenzen auf die Lebenswelt und religiöse Sozialisation junger Menschen aufgezeigt. Abschließend wird folgerichtig eine pluralitätsfähige religionspädagogische Kompetenz des pädagogischen Fachpersonals mit der Notwendigkeit einer reflexiven religiösen Haltung und Identität in Verbindung gebracht.

In Kapitel vier werden religiöse Bildungsbereiche und religionspädagogische Handlungsfelder einführend dargestellt (vorbereitete Umgebung, spirituelle Angebote wie Stilleübungen und Legematerialien, kindertheologische Kommunikation, Projektarbeiten mit religiösen Erfahrungsangeboten, interreligiöse und interkulturelle Öffnung im pädagogischen Alltag sowie in Bezug auf die Erziehungs- und Bildungspartnerschaft). Anschließend werden gegenwärtige religionspädagogische Ansätze vorgestellt, die analog zum bildungswissenschaftlichen Diskurs, das Kind, seine Anlagen und seinen Erfahrungsraum ins Zentrum religiöser Bildungsprozesse rücken, wie der dimensionale Ansatz nach Scheilke und Schweitzer oder die religionssensible Erziehung und Bildung nach Lechner und Gabriel aufgenommen von Weber. Daran anknüpfend erläutert Clauß Peter Sajak sein Konzept der alltagsorientierten Bildung „Religion in allen Dingen“, das religiöse Bildungsmöglichkeiten und religionspädagogische Handlungsempfehlungen ausgehend von konkreten Alltagssituationen entfaltet. 
Kapitel fünf gibt einen exemplarischen Überblick über Methoden, die für die religionspädagogische Elementarbildung relevant sind (Kinderphilosophie und Kindertheologie, Erkunden religiöser Orte, Erzählen und Gestalten von Glaubensgeschichten, musisch-ästhetische Zugänge, Entwicklung von Ritualen, Einüben von Stille und Achtsamkeit). Auch hier rücken die Autoren das Kind als handelnd-denkendes Subjekt in den Mittelpunkt methodischdidaktischer Zugänge und Konzeptionen und bringen am Beispiel narrativer Bildungsangebote orientiert am Koran eine interreligiöse Perspektive ein.

Wie schon die Vorgängerausgabe ist auch die Neubearbeitung so aufgebaut, dass in Teil A jedes Kapitels einführende Grundkenntnisse vermittelt werden, die in Teil B dann durch Reflexions- und Diskussionsanregungen sowie didaktisch-methodische Impulse vertieft werden können. Diese Struktur erscheint grundsätzlich sinnvoll, insbesondere auch, weil die vorgeschlagenen Übungen und Reflexionsformate durchaus offen für inter- und intrareligiöse Diversität sind und den angehenden Erzieher*innen ein insgesamt hohes Maß an Reflexions- und Kommunikationskompetenz zu vermitteln suchen. Allerdings stellt sich die Frage nach einer verstärkten Demokratisierung von Lern- und Bildungsprozessen, sowohl innerhalb der Ausbildung als auch in der pädagogischen Arbeit mit den Kindern. Zwar wird die Wertschätzung von Vielfalt und individuellen Zugängen an vielen Stellen des Arbeitsbuches deutlich, die Selbststeuerung von Bildungsprozessen innerhalb einer anregenden Lernlandschaft, die durch eine intensive Begleitung der individuellen Lernprozesse seitens der Erzieher*innen bzw. der Berufsschullehrkräfte erfolgen könnte, wird jedoch durch die jeweils vorgeschlagenen Impulse nicht erreicht.

Zusammenfassend bietet das Arbeitsbuch eine gelungene Zusammenstellung von Grundlagenwissen, Reflexionsübungen und methodisch-didaktischen Kenntnissen, die die Kompetenzbereiche der religionspädagogischen Ausbildung abbilden und integriert stellenweise auch eine inter- und intrareligiöse Perspektive. Inwieweit die Vorschläge zur methodisch-didaktischen Umsetzung in den Ausbildungsschulen und Einrichtungen, unter Einbezug und Beteiligung der Adressaten, tatsächlich diversitätssensibel und subjektorientiert umgesetzt werden, kann an dieser Stelle nicht geklärt werden. Letztlich gibt das Arbeitsbuch wichtige Impulse, die jedoch vor Ort situationsorientiert und prozessbegleitend modifiziert und ergänzt werden müssen. Dies gelingt in Bildungsprozessen, die von Beteiligungs- und Teilhabestrukturen gekennzeichnet sind. 